\title{
Density matrix renormalization group study of conjugated polymers with transverse $\pi$-conjugation
}

\author{
Y. Yan and S. Mazumdar ${ }^{1}$ \\ ${ }^{1}$ Department of Physics, University of Arizona, Tucson, AZ 85721
}

(Dated: today)

\begin{abstract}
We report accurate numerical studies of excited state orderings in long hypothetical $\pi$-conjugated oligomers in which the hydrogen atoms of trans-polyacetylene are replaced with conjugated sidegroups, within modified Hubbard models. There exists a range of the bare Coulomb repulsion for which the excited state ordering is conducive to photoluminescence in the substituted systems, even as this ordering is opposite in the unsubstituted polyenes of the same lengths. Our work provides motivation to study real $\pi$-conjugated polymers with transverse conjugation and small optical gaps.
\end{abstract}

PACS numbers: 42.70.Jk, 71.20.Rv, 71.35.-y, 78.30.Jw

Materials that luminesce in the infrared are of interest because of their potential application in infrared lasers for telecommunications. Designing $\pi$-conjugated polymers that can emit light in the infrared with high quantum efficiency (QE) is an intriguing scientific problem, as all known organic emissive systems with strong photoluminescence (PL) emit either in the visible or ultraviolet. Trans-polyacetylene (t-PA) with absorption threshold at $1.6 \mathrm{eV}$, for example, is nonluminescent. This is a consequence of the Coulomb correlation between $\pi$-electrons, which drives the lowest two-photon state, the $2^{1} \mathrm{~A}_{g}$, below the one-photon optical exciton ${ }^{1}$, the $1^{1} \mathrm{~B}_{u}$. The optically pumped $1^{1} \mathrm{~B}_{u}$ in this case decays in ultrafast timescale to the $2^{1} \mathrm{~A}_{g}$, radiative transition from which is forbidden. Although single-walled carbon nanotubes (SWNTs) absorb in the infrared, their PL is extremely weak $^{2}$. Theoretical work have found forbidden dark excitons below the optical exciton in the $\mathrm{SWNTs}^{3.4}$, and it has been suggested that the reason for the weak PL in these systems is same as in t-PA ${ }^{3}$. Strong PL in emissive $\pi$-conjugated polymers such as poly-paraphenylene (PPP) and poly-paraphenylenevinylene (PPV) is due to excited state ordering $\mathrm{E}\left(2^{1} \mathrm{~A}_{g}\right)>\mathrm{E}\left(1^{1} \mathrm{~B}_{u}\right)$ in these systems [where $\mathrm{E}(\ldots)$ is the energy of the state]. This reversed excited state ordering (with respect to t-PA) is due to increased molecular exciton character of the wavefunctions, which leads to confinement of the particle and the hole in the excited $1^{1} \mathrm{~B}_{u}$ state along the backbone of the polymer. The confinement, as well as the reversed excited state ordering are reproduced within effective linear chain models for the emissive $\pi$-conjugated polymers, within which the backbone phenyl groups are modeled by $\mathrm{C}-\mathrm{C}$ bonds much stronger than the standard "double" bond of t-PA ${ }^{5,6}$. Since confinement increases both $\mathrm{E}\left(1^{1} \mathrm{~B}_{u}\right)$ and $\mathrm{E}\left(2^{1} \mathrm{~A}_{g}\right)$, it is clear that the standard prescription for light emission from $\pi$-conjugated polymers, viz., increasing effective bond alternation ${ }^{5.6}$, cannot lead to materials that emit in the infrared.

Mazumdar et al. have suggested that simultaneous small optical gap and $\mathrm{E}\left(2^{1} \mathrm{~A}_{g}\right)>\mathrm{E}\left(1^{1} \mathrm{~B}_{u}\right)$ can be obtained by substituting the hydrogen atoms of t-PA with conjugated side groups ${ }^{7.8}$, a process that has been re- ferred to as "site-substitution". The original goal here was to obtain a theoretical understanding of the high QE of the PL in polydiphenylacetylenes (PDPAs) ${ }^{9.10}$. The absorption and emission wavelengths of the experimental PDPA systems are in the visible, but this is due to their finite conjugation lengths $\frac{11}{11}$. On the other hand, the experimental demonstration that PL in PDPAs is from the backbone PA chain and not the trans-stilbene molecular unit ${ }^{10}$ confirmed the reversed excited state ordering in the PDPAs. Theoretically, it was suggested that the phenyl substituents led to electron delocalization away from the backbone $\mathrm{C}$ atoms into the transverse substituent groups, causing a reduced effective Hubbard repulsion between two $\pi$-electrons occupying the same backbone $\mathrm{C}$ atom $^{7}$. The smaller effective Hubbard repulsion, in turn, simultaneously lowered $\mathrm{E}\left(1^{1} \mathrm{~B}_{u}\right)$ and raised the relative $\mathrm{E}\left(2^{1} \mathrm{~A}_{g}\right)$. Alternatively, it was also shown from explicit calculations that site-substitution caused not only transverse delocalization but also simultaneous longitudinal confinement of the particle and the hole along the backbone in the excited $1^{1} \mathrm{~B}_{u}$ state ${ }^{8}$. The longitudinal particle-hole confinement then gives the reversed excited state ordering, exactly as in polymers with large optical gaps ${ }^{5.6}$.

Direct demonstration of $\mathrm{E}\left(2^{1} \mathrm{~A}_{g}\right)>\mathrm{E}\left(1^{1} \mathrm{~B}_{u}\right)$ in the $\mathrm{PDPAs}$ requires high order configuration interaction (CI) calculation that is difficult because of the large number of $\mathrm{C}$ atoms in the unit cell $\frac{8}{}$. Dallakyan et al $\frac{12}{2}$ have therefore performed "proof-of-concept" calculations for a hypothetical prototype polymer, polydiethylenepolyacetylene (system [1] in Fig. 1), suggesting that PDPAs and other more complex systems can be modeled by [1]. Correlated electron calculations for [1] are simpler than for PDPAs due to the smaller number of $\mathrm{C}$ atoms in the former. The number of $\mathrm{C}$ atoms even in [1], however, increases rapidly with increasing number of unit cells. Exact or full CI (FCI) calculations are possible only for the two-unit oligomer of [1], and even quadruple CI (QCI) calculations become impossible for more than three units. Calculations in reference 12 were therefore performed within an approximate exciton mode ${ }^{13}$, within which only the highest occupied and lowest unoc- 


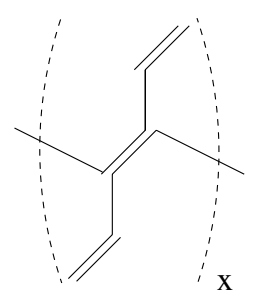

1

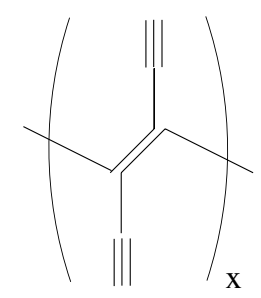

2

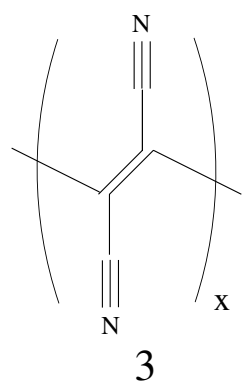

3
FIG. 1: Model hypothetical polymers with conjugated side groups whose excited state orderings are of interest.

cupied molecular orbitals (HOMO and LUMO) of each hexatriene unit of [1] were retained. This approximation allowed QCI calculations for up to five units of [1], and it was found within dimerized Hubbard model calculations with an average hopping integral of $2.4 \mathrm{eV}$ that the critical "Hubbard $U$ " (hereafter $\left.U_{c}\right)$ at which $\mathrm{E}\left(2^{1} \mathrm{~A}_{g}\right)$ becomes smaller than $\mathrm{E}\left(1^{1} \mathrm{~B}_{u}\right)$ (starting from $U=0$ ) is larger by $\sim 1 \mathrm{eV}$ in [1] than in the unsubtituted polyene of the same length. The larger $U_{c}$ in [1] is sufficient as "proof-of-concept", as this indicates that there exists a range of values for the bare Coulomb repulsion for which $\mathrm{E}\left(2^{1} \mathrm{~A}_{g}\right)>\mathrm{E}\left(1^{1} \mathrm{~B}_{u}\right)$ in the substituted oligomer even as the opposite excited state ordering occurs in the unsubstituted system. In principle, therefore, attaching side groups that are more extended than in [1] can lead to systems with excited state ordering conducive to light emission.

The approximate nature of the exciton basis approach as well as the severe chain length limitations are the obvious limitations of the previous calculations 12 . More importantly, the verification of the original idea that enahanced particle-hole delocalization into the sidegroups lead to confinement along the backbone ${ }^{\frac{8}{\underline{ }}}$ requires that the computational technique be able to distinguish between polymer [1] and the slightly modified hypothetical polymers [2] and [3], also included in Fig. 1. Within the proposed mechanism of reference 8 , for instance, the larger hopping integral corresponding to the triple bond in the side group in [2] should lead to a $U_{c}$ even larger than in [1]. Attaching an electron attracting CN group, as in [3], should decrease the electron density on the backbone $\mathrm{C}$ atoms, and enhance $U_{c}$ even more strongly ${ }^{8}$. There is unfortunately no way to test these ideas precisely within the exciton basis approach, as the structural modifications in [2] and [3], with respect to [1], change the HOMO and the LUMO of the unit cell weakly, with the changes distributed evenly over the other MOs.

In the present paper, we report the results of density matrix renormalization group (DMRG) calculations of $U_{c}$ for all three systems [1], [2] and [3]. The DMRG is a highly accurate numerical technique for correlated electron calculations for long one dimensional systems $\underline{14}$ that has been extensively applied to determine the electronic structures of $\pi$-conjugated polymers ${ }^{15.16}$. In order to investigate excited states, we use the symmetrized DMRG (SDMRG) technique of Ramasesha et al ${ }^{15}$, which allows the targeting of the lowest states of a given symmetry subspace. In our calculations we exploit spatial, electronhole (for [1] and [2]) and spin parity symmetries. Our wavefunctions are eigenstates of the total z-component of the spin, $\mathrm{S}_{z}$ and not the total spin $\mathrm{S}$. This presents no problems in identifying the $1^{1} \mathrm{~A}_{g}$ and the $1^{1} \mathrm{~B}_{u}$, which are the lowest states with specific spatial and electronhole symmetries and total spin $S=0$. In the case of the $2^{1} \mathrm{~A}_{g}$, however, care should be taken that the target $\mathrm{S}_{z}$ $=0$ state does not belong to any of the $\mathrm{S}>0$ states. We rule out the possibility of identifying an incorrect excited state as the $2^{1} \mathrm{~A}_{g}$ by explicitly calculating the transition dipole moment between the target state and the $1{ }^{1} \mathrm{~B}_{u}$ : dipole moments are nonzero only between states with the same total spin. The SDMRG technique allows the determination of the energies of the $1^{1} \mathrm{~A}_{g}$, the $1^{1} \mathrm{~B}_{u}$ and the $2^{1} \mathrm{~A}_{g}$ with high accuracy upto 12 units (see below). We confirm that within the dimerized Hubbard model, $U_{c}$ for [1] is indeed larger than in the unsubstituted polyene of the same chain length, as claimed before ${ }^{12}$, although the difference is smaller than in the previous approximate calculation. More importantly, though, the $U_{c}$ for [2] is substantially larger, while that for [3] is larger still, confirming our original hypothesis. We postpone discussions of the implications of these results for real $\pi$-conjugated polymers until later.

As in reference 12, we consider the modified Hubbard Hamiltonian,

$$
\begin{array}{r}
H=H_{1 e}+H_{e e} \\
H_{1 e}=\sum_{i} \epsilon_{i} n_{i}-\sum_{\langle i j\rangle, \sigma} t_{i j} c_{i, \sigma}^{\dagger} c_{j, \sigma} \\
H_{e e}=U \sum_{i} n_{i, \uparrow} n_{i, \downarrow}
\end{array}
$$

In the above, $c_{i, \sigma}^{\dagger}$ creates a $\pi$-electron of spin $\sigma$ on site $i, n_{i, \sigma}$ is the number of electrons with spin $\sigma$ on site $i$, and $\langle.$.$\rangle implies nearest neighbors. H_{1 e}$ describes the oneelectron site energies and the nearest neighbor hopping of electrons, and $H_{e e}$ consists of the electron-electron (e-e) interaction within the Hubbard approximation. The hopping integrals $t_{i j}$ are taken to be $t_{1}=2.4(1-\delta) \mathrm{eV}$ and $t_{2}$ $=2.4(1+\delta) \mathrm{eV}$, with $\delta=0.07$, corresponding to single and double bonds, respectively. For the triple bonds in [2] and [3] we choose $t_{i j}=3 \mathrm{eV}$. These values of $t_{i j}$ are considered standard for $\pi$-conjugated systems within correlated electron models 17 . We have ignored all Coulomb interactions other than the on-site repulsion $U$, since the $2 \mathrm{~A}_{g}-1 \mathrm{~B}_{u}$ crossover is related to this interaction only, with the spin-independent long range intersite Coulomb interactions merely modifying the absolute magnitude of $U$ at which the crossover occurs. For the carbon-only systems [1] and [2] in Fig. 1 we take all site energies $\epsilon_{i}$ $=0$. For system [3], we have chosen the standard site 


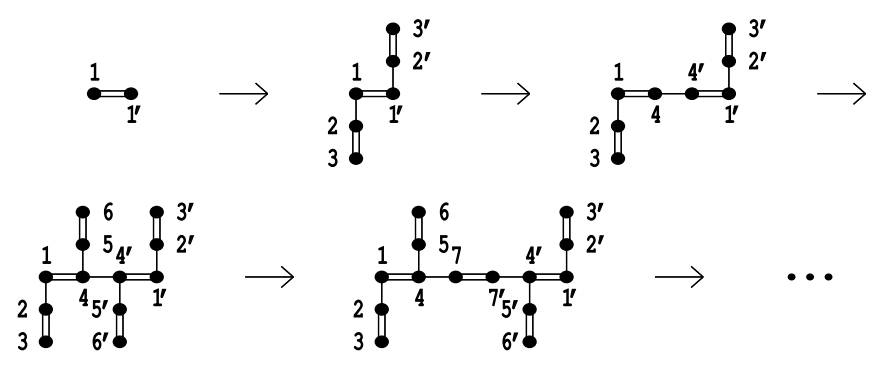

FIG. 2: Schematic diagram of the building up of the substituted polymer [1].

energy of $-3.0 \mathrm{eV}$ for the nitrogen atoms ${ }^{18}$. In principle, we should have also chosen a different Hubbard $U$ for the nitrogen atoms, but since this difference is quite small18, and since our numerical procedure consists of varying the Hubbard interaction, we have chosen the same $U$ for both carbon and nitrogen.

The DMRG scheme that we use to calculate long chain behavior of the substituted polymers of Fig. 1 is shown in Fig. 2. We add two atoms at each step of the DMRG procedure. This approach proved to be better than adding single atoms at each step.

We have checked the accuracy of our calculations by comparing the DMRG energies at $U=0$ of all three eigenstates we investigated with their exact Hückel energies. In Table 1, we have listed the DMRG and Hückel energies for the $1^{1} \mathrm{~A}_{g}$, the $1^{1} \mathrm{~B}_{u}$ and the $2^{1} \mathrm{~A}_{g}$ for the 6 and 12 -unit oligomers of [1] , and the 6 -unit oligomers of [2] and [3], respectively. We find a high level of accuracy in all three absolute energies. The excitation energies are slightly overestimated, with the overestimation of $\mathrm{E}\left(2^{1} \mathrm{~A}_{g}\right)$ being slightly larger than that for $\mathrm{E}\left(1^{1} \mathrm{~B}_{u}\right)$ (for e.g., the overestimation for $\mathrm{E}\left(2^{1} \mathrm{~A}_{g}\right)$ of [3] is $4 \%$ while that for $\mathrm{E}\left(1^{1} \mathrm{~B}_{u}\right)$ is $1.2 \%$.) We comment on this aspect following the presentation of the numerical results, where we show that this does not change our principal conclusion. Here we emphasize that DMRG energies are least accurate in the $U=0$ limit, where all wavefunctions are delocalized, and the accuracy improves with increasing localization at larger $U$.

TABLE I: Comparison of DMRG energies at $U=0$ with $\mathrm{H} /$ "uckel energies (in units of $|t|$ )

\begin{tabular}{|c|c|c|c|c|c|}
\hline Polymer & $\begin{array}{l}\text { Number } \\
\text { of Units }\end{array}$ & Method & $1 \mathrm{Ag}$ & $1 B u$ & $2 A g$ \\
\hline \multirow[b]{2}{*}{ [1] } & \multirow[t]{2}{*}{ 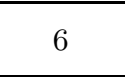 } & DMRG & -45.31856 & -44.94023 & -44.72728 \\
\hline & & Huckel & -45.31860 & -44.94138 & -44.73250 \\
\hline \multirow[t]{2}{*}{$\begin{array}{llll}1 & \end{array}$} & \multirow[b]{2}{*}{12} & DMRG & -91.08550 & -90.82209 & -90.69684 \\
\hline & & Huckel & -91.08582 & -90.82938 & -90.72156 \\
\hline \multirow{2}{*}{ [2] } & & $\overline{D M R G}$ & -49.25526 & -48.82161 & -48.56106 \\
\hline & & & -49.25548 & -48.82795 & -48.58643 \\
\hline \multirow[b]{2}{*}{ [3] } & & DMRG & -67.95981 & -67.52728 & -67.29174 \\
\hline & & Huckel & -67.96001 & -67.53034 & -67.31854 \\
\hline
\end{tabular}

In Figs. 3(a) and (b) we have plotted the calculated DMRG excitation energies $\mathrm{E}\left(1^{1} \mathrm{~B}_{u}\right)$ and $\mathrm{E}\left(2^{1} \mathrm{~A}_{g}\right)$ with
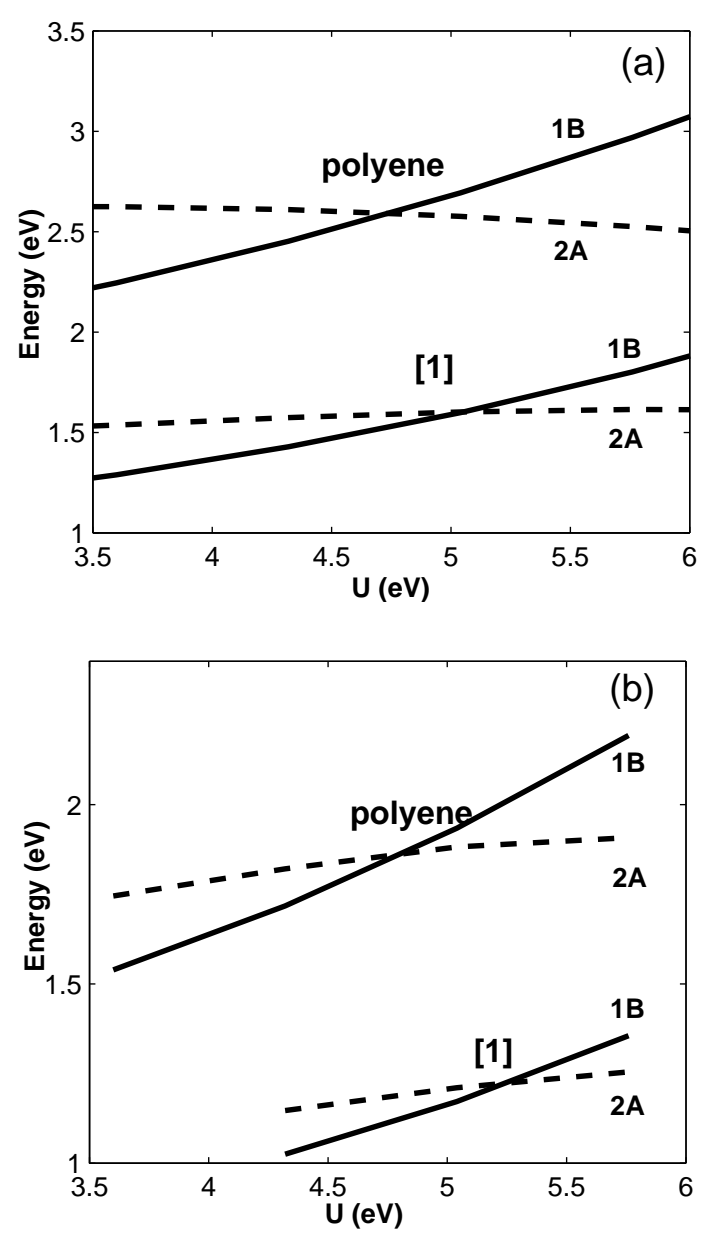

FIG. 3: (a) The excitation energies $\mathrm{E}\left(1^{1} \mathrm{~B}_{u}\right)$ and $\mathrm{E}\left(2^{1} \mathrm{~A}_{g}\right)$ for the unsubstituted polyene and the substituted system [1] of Fig. 1 for (a) 6-unit, and (b) 12-unit oligomers.

respect to the ground state energy for the 6- and 12 unit unsubstituted polyenes and oligomers of [1]. These results are to be compared with the approximate results for the 4 - and 5-unit oligomers ${ }^{12}$. As in reference [12], the excitation energies in the substituted polyenes are lower than the unsubstituted systems. The initial increase in $\mathrm{E}\left(2^{1} \mathrm{~A}_{g}\right)$ with $U$ was seen also in reference [12], as well as in earlier DMRG calculations of long unsubstituted polyenes $\frac{15}{15}$, where it was ascribed to the small $2^{1} \mathrm{~A}_{g}$ $1^{1} \mathrm{~B}_{u}$ gap at $U=0$ in long chains. The increase persists in the 12-unit oligomers for the range of $U$ we have studied, as the $U=02^{1} \mathrm{~A}_{g}-1^{1} \mathrm{~B}_{u}$ gap is quite small at this chain length. The critical $U_{c}$ at which $\mathrm{E}\left(2^{1} \mathrm{~A}_{g}\right)<\mathrm{E}\left(1^{1} \mathrm{~B}_{u}\right)$ is indeed larger in the substituted polyene [1] than in the unsubstituted polyene, as claimed befor 12 . However, the difference in the $U_{c}$ is $\sim 0.5 \mathrm{eV}$ as opposed to the $\sim 1.0$ $\mathrm{eV}$ found previously.

The critical $U_{c}$ are even larger in the oligomers of [2] and [3], as seen in Fig. 4, where we have plotted $\mathrm{E}\left(1^{1} \mathrm{~B}_{u}\right)$ and $\mathrm{E}\left(2^{1} \mathrm{~A}_{g}\right)$ for all three systems of Fig. 1. Detailed examination of the different behavior of the three systems provides indirect proof of our claim that site-substitution 
reduces the effective Hubbard repulsion on the backbone $\mathrm{C}$ atoms. In particular, we draw attention to the qualitatively different behavior of [2] and [3]. Both $\mathrm{E}\left(1^{1} \mathrm{~B}_{u}\right)$ and $\mathrm{E}\left(2^{1} \mathrm{~A}_{g}\right)$ are larger in [2], relative to [1], but for a given $U$, the increase in $\mathrm{E}\left(2^{1} \mathrm{~A}_{g}\right)$ is larger than that in $\mathrm{E}\left(1^{1} \mathrm{~B}_{u}\right)$. This is the reason for larger $U_{c}$ in [2]. This feature is qualitatively similar to the behavior of these two energy gaps in effective linear chains as the bond alternation is increased ${ }^{5.6}$. The behavior of [3] is, however, completely different. $\mathrm{E}\left(2^{1} \mathrm{~A}_{g}\right)$ of [3], exactly as that of [2], is larger than that of [1] at all $U \cdot \mathrm{E}\left(1^{1} \mathrm{~B}_{u}\right)$ of [3], on the other hand, is larger than that of [1] at $U=0$ but smaller than that of [1] for $U>4.5 \mathrm{eV}$. It is precisely this feature of [3]. i.e., relatively small $\mathrm{E}\left(1^{1} \mathrm{~B}_{u}\right)$ and large $\mathrm{E}\left(2^{1} \mathrm{~A}_{g}\right)$ at larger $U$ that gives the largest $U_{c}$ for this system. Such reduction of the optical gap with heteroatom substitution has also been observed in other theoretical work ${ }^{18.19}$, but the mechanism of the optical gap reduction here is quite different. This behavior is precisely what is expected from the effective Hubbard repulsion mode ${ }^{7}$. At small $U$ the optical gap is determined by bond and site alternations, and $\mathrm{E}\left(1^{1} \mathrm{~B}_{u}\right)$ in [3] is larger than that of [1] and is close to that of [2] (see Table 1). At moderate to large $U$ the ground state is predominantly covalent with all backbone $\mathrm{C}$ atoms singly occupied. The $1^{1} \mathrm{~B}_{u}$ is ionic, with one vacant and one doubly occupied $\mathrm{C}$ atom each. With electron attracting sidegroups, the "double occupancy" in the $1^{1} \mathrm{~B}_{u}$ is delocalized into the sidegroups, reducing the effective Hubbard repulsion, and consequently the optical gap. The $2^{1} \mathrm{~A}_{g}$ is covalent at large $U$, and hence its energy is unaffected (notice that the difference between the $\mathrm{E}\left(2^{1} \mathrm{~A}_{g}\right)$ in [3] and [1] continues to be the same at small and large $U$ in Fig. 4). Coming back now to the issue of overestimation of energies within the DMRG procedure. $U_{c}$ for [3] is so much larger than that of [1] that even if the extent of overestimation at $U=0$ is assumed to persist at $U \neq 0$, and even if this overestimation occurs only with $\mathrm{E}\left(2^{1} \mathrm{~A}_{g}\right)$ and not $\mathrm{E}\left(1^{1} \mathrm{~B}_{u}\right)$ (in practice neither statement is true), our statement regarding strong enhancement of $U_{c}$ by substitution with electron attracting sidegroups continues to be valid.

In conclusion, we have shown from accurate DMRG calculations that site substitution with $\pi$-conjugated sidegroups can indeed simultaneously lower the optical gap and give excited state ordering that is conducive the light emission. This is particularly true when the con- jugated sidegroup substituent is electron attracting. Although the present work involves hypothetical structures, we believe that they provide the motivation for examination of the excited state ordering as well as the PL behavior of real "small bandgap" polymers with transverse $\pi$-conjugation, such as polyisothianaphthene and poly(isonaphthothiophene), with optical gaps of $1.1 \mathrm{eV}^{20}$ and $1.5 \mathrm{eV}^{21}$, respectively. In contrast to the very intense studies of the wider optical gap polymers, these small gap polymers have received much less attention. Good sample quality has also been elusive. Theoreti-

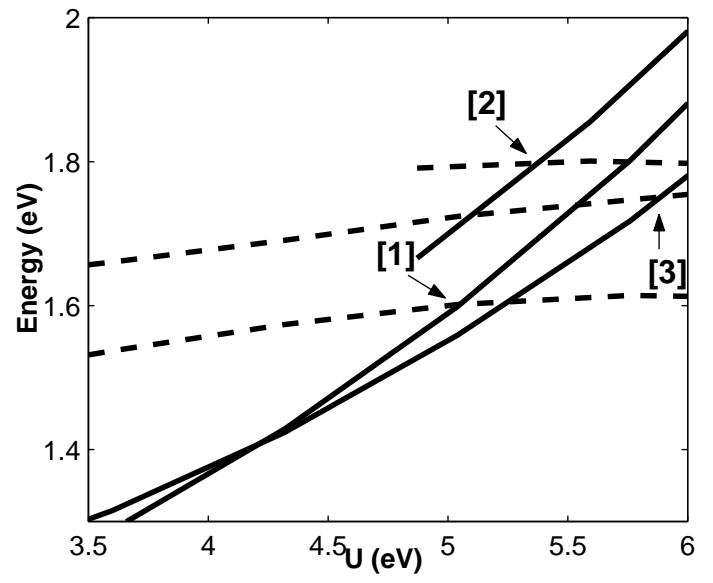

FIG. 4: The excitation energies $\mathrm{E}\left(1^{1} \mathrm{~B}_{u}\right)$ (solid curves) and $\mathrm{E}\left(2^{1} \mathrm{~A}_{g}\right)$ (dashed curves) for 6-unit oligomers of all three substituted systems of Fig. 1.

cal work on these systems until now have focused on explaining the small optical gaps and the competition between benzenoid and quinoid structures within oneelectron theories 22,23 . Whether or not the observed small optical gaps can be explained within many-electron theory, that is now established for the wider gap polymers, is clearly of interest. We are currently pursuing theoretical studies of these systems within the DMRG procedure to understand the consequence of transverse $\pi$-conjugation on their optical gaps and excited state ordering.

This work was partially supported by NSF-DMR0406604. We acknowledge many useful discussions on the DMRG procedure with S. Ramasesha from the I.I.Sc., Bangalore.
1 B.S. Hudson, B.E. Kohler and K.S. Schulten, in Excited States, edited by E.C. Lim (Academic Press, 1982), pp. 1 $-95$.

2 M.J. O'Connell et al., Science 297, 593 (2002). S. Lebedkin et al., J. Phys. Chem. B 107, 1949 (2003). F. Wang et al., Phys. Rev. Lett. 92, 177401 (2004). C.-X. Sheng et al., Phys. Rev. B

${ }^{3}$ H. Zhao and S. Mazumdar, Phys. Rev. Lett. 93, 157402
(2004)

4 V. Perebeinos, J. Tersoff and P. Avouris, Phys. Rev. Lett. 92, 257402 (2004).

5 Z.G. Soos, S. Etemad, D.S. Galvão, and S. Ramasesha, Chem. Phys. Lett. 194, 341 (1992).

6 Z.G. Soos, S. Ramasesha, and D.S. Galvão, Phys. Rev. Lett. 71, 1609 (1993).

7 A. Shukla and S. Mazumdar, Phys. Rev. Lett. 83, 3944 
(1999).

8 H. Ghosh, A. Shukla and S. Mazumdar Phys. Rev. B 62, 12763 (2000).

9 I. Gontia et al. Phys. Rev. Lett. 82, 4058 (1999).

10 R. Hidayat et al., Phys. Rev. B 61, 10167 (2000).

11 K. Tada et al., Proc. SPIE-Int. Soc. Opt. Eng. 3145, 171 (1997).

12 S. Dallakyan, M. Chandross and S. Mazumdar, Phys. Rev. B 68, 075204 (2003).

13 M. Chandross, Y. Shimoi and S. Mazumdar, Phys. Rev. B 59, 4822 (1999).

14 S.R. White, Phys. Rev. Lett. 69, 2863 (1992). Phys. Rev. B 4810345 (1993).

15 S. Ramasesha, S.K. Pati, Z. Shuai and J.L. Brédas, Adv. Quant. Chem. 38, 121 (2001).
16 W. Barford, Electronic and Optical Properties of Conjugated Polymers, Clarendon (Oxford, 2005).

17 D. Baeriswyl, D.K. Campbell, and S. Mazumdar, in Conjugated Conducting Polymers, edited by H. Kiess, Springer Verlag, Berlin (1992).

18 I.D.L. Albert, S. Ramasesha and P.K. Das, Phys. Rev. B 43, 7013 (1991).

19 Y.S. Lee and M. Kertesz, J. Chem. Phys. 88, 2609 (1988).

20 M. Kobayashi et al., J. Chem. Phys. 82, 5717 (1985).

21 Y. Ikenoue, Synth. Metals 35, 263 (1990).

22 Y.S. Lee, M. Kertesz, and R. Elsenbaumer, Chem. Mater., 2, 526 (1990).

23 J. Kürti, P.R. Surjan and M. Kertesz, J. Am. Chem. Soc. 113, 9865 (1991). 